\title{
Nephrotoxicity in patients receiving immune checkpoint inhibitors
}

\author{
Teresa Chuva
}

Nephrology Department, Instituto Português de Oncologia do Porto

\section{ABSTRACT}

Over the last years, immunotherapy has become part of the most important strategies to treat some types of cancer. Immune checkpoint inhibitors are within this new class of drugs and consist of monoclonal antibodies that target inhibitory receptors expressed on T cells. While revolutionary results have been achieved with these therapies, their widespread use has also brought to light multiple immune-related adverse effects that impact the patient's quality of life and survival, posing new challenges to clinicians. The kidney is one of the affected organs, and nephrotoxicity has probably been underestimated in the first clinical trials. The etiopathogenic mechanisms involved and the management of renal disease in this context are not fully known. This review aims to illustrate the most recent data on the clinical presentation, diagnosis, treatment, and behavior of patients with kidney complications of immune checkpoint inhibitors, including particularly vulnerable subjects, such as transplant recipients. A growing number of patients are being handled with these drugs, and nephrologists are expected to be part of the multidisciplinary approach required by the new immunotherapies.

Key-words: Immune checkpoint inhibitors, nephrotoxicity, immune-related adverse events, acute kidney injury, interstitial nephritis, immunotherapy, onconephrology

\section{INTRODUCTION}

The treatment of cancer has seen dramatic changes over the last decades, thanks to an increased molecular understanding of its development. Immunotherapy has recently brought a whole new paradigm, establishing a revolutionary principle by exploring and stimulating the ability of our immune system to attack tumor cells. Immune checkpoint inhibitors (ICPI) are among the new therapeutic families, and their use has been multiplying, with numerous ongoing clinical trials and their application expanding from palliative to adjuvant and neoadjuvant treatment of cancer, administered in multiple different combinations, rapidly increasing the population exposed to the new immunomodulators ${ }^{1,2}$.

ICPI are monoclonal antibodies that block immunosuppressor receptors and their ligands, such as programmed cell death protein 1 (PD-1), programmed cell death protein-ligand 1 (PDL-1), and cytotoxic $T$ lymphocyte-associated antigen $4(\mathrm{CTLA}-4)^{3}$. These are inhibitory molecules used by tumor cells to evade immunologic responses of $T$ cells, inducing immune tolerance. CTLA-4 and PD-1 are receptors expressed on the surface of T cells that bind to their ligands, CD80/CD86, and

\section{Table 1}

ICPI approved by Food and Drug Administration

\begin{tabular}{c|c|c} 
Anti-CTLA-4 & anti-PD-1 & anti-PDL-1 \\
\hline ipilimumab & pembrolizumab & atezolizumab \\
& nivolumab & avelumab \\
& cemiplimab & durvalumab
\end{tabular}

CTLA-4: cytotoxic T lymphocyte-associated antigen 4; PD-1: Programmed cell death protein 1; PDL-1: programmed cell death protein-ligand 1
PD-L1, respectively. While CTLA-4 inhibits T-cell activation at a proximal step in the immune response, PD-1 attenuates T-cell activation at later stages in peripheral tissues ${ }^{4,5}$. ICPI act by preventing the receptors and ligands from interacting with each other, with this relieving T-cells suppression and mediating an anti-tumor immune response. Current ICPI approved by Food and Drug Administration are exhibited in Table 1. The CTLA-4 inhibitor ipilimumab was the first of its kind.

\section{IMMUNE-RELATED ADVERSE EVENTS}

Activating the immune system to fight cancer comprises specific risks. While we aim at tumor cells, healthy tissues may also be unintentionally targeted, triggering autoimmune (Al) responses that might affect all organs. Since the ability to control the immune system is still being refined, clinicians that deal with cancer patients should be aware of the potential immune-related adverse events (irAEs), which may affect $>50 \%$ of patients on immunotherapy ${ }^{6}$. The skin seems to be one of the most affected organs, with more than one-third of patients experiencing a maculopapular rash ${ }^{7}$. Gastrointestinal symptoms, such as diarrhea and sometimes severe colitis, may be seen in $1-25 \%$ patients ${ }^{8}$ and endocrine irAEs, like hypophysitis or thyroid dysfunction, have been reported in $1-8 \%$ patients $^{9}$. Organ-specific toxicities seem to differ slightly between anti-PD-1 and anti-CTLA-4 inhibitors, yet it is still not fully understood why this happens. The different expression of the immunologic receptors in distinct organs might determine distinct responses ${ }^{6}$.

The role of immune checkpoints has been proved in animal models. Mice lacking CTLA-4 die from aggressive lymphoproliferative diseases ${ }^{10}$, while mice deficient in PD-1 have a more limited and variable Al response, developing diseases such as Systemic Lupus Erythematosus ${ }^{11}$. Immune checkpoints have also been explored in clinical 
practice. Treatment with the CTLA-4-like CD80/86 ligand abatacept, which has precisely the opposite effect of ipilimumab, has been recommended for several Al diseases ${ }^{12-14}$.

Similar to what is observed with animal models, when compared to ipilimumab, the newer anti-PD-1/PD-L1 inhibitors seem to offer greater safety ${ }^{15}$, which could be explained by the less specific effect of CTLA-4 inhibition on T cell activation ${ }^{16}$. The combined use of CTLA-4 and PD-1 blockade is associated with a higher risk of irAEs but may result in relevant clinical synergism and survival benefit ${ }^{17,18}$.

Diverse mechanisms have been proposed to understand the physiopathology of irAEs: 1) cross-reactivity may occur when T-cells increase their activity against tumoral antigens that might resemble auto-antigens expressed on healthy tissues ${ }^{19}$; 2) PD-1/PD-L1 appear to play a role in modulating humoral immunity, helping to maintain self-tolerance ${ }^{20}$; ICPI might disrupt an immunologic equilibrium, increasing auto-antibodies that were previously not pathologic; 3) elevated levels of inflammatory cytokines may contribute to the lesions seen with ICPI, such as podocyte foot process effacement seen with podocytopathies ${ }^{21,22}$; 4) complement-mediated inflammation due to direct binding of an ICPI to its receptor expressed on normal tissue can contribute to aggravate the lesions on healthy tissues ${ }^{6}$.

\section{RENAL DAMAGE}

The kidney is one of the multiple organs affected by ICPI. First trials estimated an overall incidence of acute kidney injury (AKI) of 2.2\%, with increased frequency when the combination ipilimumab/nivolumab was used $(4.9 \%)^{2}$. However, with the widespread use of ICPI, AKI has been estimated to be as high as $29 \%{ }^{23}$. As the population on immunotherapy grows and diversifies, a more extensive range of toxicities has been unfolding.

Different grading systems limit the reports on AKI since the Common Terminology Criteria for Adverse Events (CTCAE) used to classify nephrotoxicity in cancer patients differs from the highly validated KDIGO criteria. The former does not consider lower grade kidney injury as defined by the

\section{Table 2}

Classifications of AKI: Comparison of KDIGO and CTCAE v5.0

\begin{tabular}{|c|c|c|}
\hline Stages & KDIGO & CTCAE v5.0 \\
\hline 1 & $\begin{array}{c}\text { Scr } 1.5-1.9 \text { times } x \text { baseline } \\
\text { or } \\
\geq 0.3 \mathrm{mg} / \mathrm{dL} \text { increase }\end{array}$ & Scr >ULN to $1.5 \times$ ULN \\
\hline 2 & Scr 2-2.9 times $x$ baseline & Scr $>1.5-3 \times$ baseline or $1.5-3 \times$ ULN \\
\hline 3 & $\begin{array}{c}\text { Scr } 3 \text { times } x \text { baseline } \\
\text { or } \\
\text { Increase in serum creatinine } \\
\text { to } \geq 4 \mathrm{mg} / \mathrm{dL} \\
\text { or } \\
\text { Initiation of renal replacement } \\
\text { therapy }\end{array}$ & Scr $>3 \times$ baseline or $3-6 \times$ ULN \\
\hline 4 & & $\mathrm{Scr}>6 \times$ ULN \\
\hline
\end{tabular}

AKI - acute kidney injury; KDIGO - Kidney Disease: Improving Global Outcomes; CTCAE - Common Terminology Criteria for Adverse Events; Scr - serum creatinine; ULN - upper limit of normal
KDIGO criteria (Table 2). Another aspect that prevents an adequate report on immunotherapy nephrotoxicity is the fact that urinary samples are frequently omitted at first evaluations, which makes interpretations of altered urinary tests difficult after starting therapy, considering that kidney complications are common in cancer patients ${ }^{24,25}$. Hence, the true incidence of kidney involvement seen with ICPI is probably underestimated.

\section{Acute tubulointerstitial nephritis}

The most common kidney manifestation seen with ICPI is AKI due to acute tubulointerstitial nephritis (ATIN), which seems to be present most of the time, even when other renal complications develop, such as vasculitis or glomerular injury 22,26 . However, ATIN, in this context, differs from the typical drug-induced reaction. To start with, there is a highly variable temporal pattern: it has been described up to 22 months ${ }^{27}$ after initiation of ICPI, and there have been case reports of reactions more than 8 weeks after taking the last dose of immunotherapy ${ }^{2}$, which contrasts with the 7-10 days usually observed after the beginning of the offending drug associated with classical ATIN ${ }^{28}$.

Similarly, there isn't a clear causality between drug exposure and AKI. On the one hand, Gallan et al. describe a case in which AKI relapsed one month after stopping corticosteroids (CCT), even without resuming the offending $\mid \mathrm{IPI}^{26}$. On the other hand, some patients were repeatedly exposed to the same drug, without any further event, as demonstrated by Cortazar et al. ${ }^{2}$

It has been hypothesized that uninhibited T cells may lead to drug-induced hypersensitivity that, when a known immunogenic molecule is involved (ex. nonsteroidal anti-inflammatory drugs or proton pump inhibitors), could cause ATIN. The case presented by Koda et al. is a good illustration, with a positive drug-induced lymphocyte stimulation test for lansoprazole alerting for the importance of recognizing medications known to cause ATIN ${ }^{29}$.

This assumption, though, differs from what is observed in animal models, where the absence of immune checkpoints suffices to cause $A \operatorname{TIN}^{11}$. The latter is also evident in patients with no known exposure to conventional nephrotoxic therapies that have also developed ATIN 27,30.

Clinically, patients typically present with AKI that might sometimes be oliguric, pyuria, hematuria, and proteinuria that is usually bland $2,27,31$, such as seen in other cases of classic ATIN ${ }^{28}$. Eosinophilia is a rare finding. Other irAEs often develop before or with $\mathrm{AKI}^{2,27}$.

Renal biopsies show tubulointerstitial infiltrates and edema, with a predominance of CD3+ mononuclear T cells. Granulomas and eosinophils may be present, although they are neither sensitive nor specific for ICPI-associated ATIN 29,30,32-34. Cassol et al. have identified a particular immunohistochemistry staining pattern for PD-L1 in inflammatory and tubular epithelial cells that seems to be only found in ICPI-induced ATIN ${ }^{27}$.

\section{Other renal findings}

Glomerular lesions are less commonly found, although there has been growing literature on the subject, with reports on lupus 
nephritis, thrombotic microangiopathy, focal segmental glomerulosclerosis (FSGS), minimal change disease, immune-complex-mediated glomerulonephritis (GN), IgA nephropathy and pauci-immune $\mathrm{GN}^{26,35-42}$. Mamlouk et al. have recently published a single-center experience on the "nephrotoxicity of immune checkpoint inhibitors beyond tubulointerstitial nephritis" 22 , where they describe their findings, such as AA amyloidosis, membranous nephropathy (negative for anti-phospholipase-A2 receptor), IgA and pauci-immune GN, c3 glomerulopathy and FSGS. It is crucial to notice, however, that previous urinalyses were not available for most of the cases (11 of 16), making it hard to exclude paraneoplastic syndromes.

Renal vasculitis has also been linked to ICPI in several reports 22,26 . It is yet unknown why the same drug may be related to so many distinct renal reactions.

\section{Electrolyte disturbances}

Different electrolyte disturbances have been associated with immunotherapy. The most common is hyponatremia, which is usually secondary to hypophysitis and hypopituitarism ${ }^{23,43}$. Electrolyte disorders may relate to several other irAEs (eg., diarrhea from colitis) or concomitant clinical features of cancer itself (eg., anorexia, nausea, and vomiting), making it difficult to assess a real causal effect of ICPI. According to Manohar et al., hypocalcemia is the only that has been significantly associated with PD-1 inhibitors, although the authors couldn't find a reasonable explanation ${ }^{44}$

\section{TREATMENT STRATEGY AND KIDNEY RECOVERY}

The ESMO Clinical Practice Guidelines on the management of toxicities from immunotherapy ${ }^{45}$ propose the following approach, depending on the CTCAE staging system:

1) In case of a grade (G) 1 renal event, the physician may continue ICPI while closely monitoring creatinine values ( $1 \mathrm{x} /$ week). Nephrotoxic drugs and hydration status should be reviewed, and other causes for AKI should be excluded.

2) In case of a G2 renal toxicity, ICPI should be withheld, renal biopsy should be considered, and oral prednisolone $0.5-1 \mathrm{mg} /$ $\mathrm{kg}$ initiated if $\mathrm{AKI}$ is attributed to irAEs.

3) AKI G3 or 4 are managed as grade 2, but (methyl)prednisolone should be considered in a higher dose: $1-2 \mathrm{mg} / \mathrm{kg}$.

4) Steroid taper: begin to taper once creatinine G1; G2 severity episode: taper over 2-4 weeks; G3/4 episode: taper over $\geq 4$ weeks

5) If AKI returns to G1/baseline: reinitiate ICPI (if on steroids, only once $<10 \mathrm{mg}$ prednisolone)

The American Society of Clinical Oncology Clinical Practice Guideline for the Management of IrAEs in patients treated with $\mathrm{ICP}^{46}$ has similar recommendations but adds: if creatinine elevations persist or worsen > 3-5 days (G3) or >2-3 days (G4), consider additional immunosuppression (e.g., mycophenolate). Although the prognosis is usually favorable, with an excellent response to CCT and generally with complete recovery of the renal function, some patients show a corticoresistant or corticodependent behavior that requires other immunosuppressors, such as mycophenolate $2,22,37$. The same drug has previously been used for the treatment of classic ATIN from different etiologies, with effective results ${ }^{47}$. Other therapies have been proposed, based on positive results observed with other organ toxicities, as is the case of infliximab, usually employed in the management of colitis. Mamlouke et al. report its successful use in patients with ATIN or IgA nephropathy attributed to $\mathrm{ICPI}^{22}$. Depending on the renal toxicity, other immunosuppressors might additionally be necessary, as is the case of rituximab for the treatment of vasculitis ${ }^{22}$. However, Gallan et al. describe 3 patients with vasculitis that was successfully treated with CCT only ${ }^{26}$.

Up to this moment, CCT seem to be the mainstay of therapy for irAEs. Other immunosuppressors should be considered on a case-by-case basis. So far, no prospective trials have defined the best treatment approach, and current recommendations are based solely on expert consensus.

One of the most important questions raised when facing an irAEs is whether ICPI may be safely resumed. A few studies have addressed the safety of restarting therapy, with inconsistent results. A retrospective study with 38 patients treated with anti-PD1 or anti-PDL1 who had irAEs that required either a delay in treatment or CCTs (or both) and were later exposed to ICPI again showed that $50 \%$ had no further irAEs, $24 \%$ had a recurrence of the first event, and $26 \%$ had a different $\operatorname{irAE}^{48}$. Cortazar et al. describe 2 patients who were rechallenged with ICPI, neither of whom had other renal irAEs ${ }^{2}$. Nakatany et al. describe another case where nivolumab was restarted (while on $5 \mathrm{mg}$ methylprednisolone) with no further occurrence ${ }^{30}$. Other authors, however, had less favorable outcomes. Glutsch et al. report a patient with whom, despite altering ICPI classes, the nephrotic syndrome reappeared ${ }^{35}$. Kitchlu et al. describe another patient whose nephrotic syndrome recurred after re-exposure to ipilimumab ${ }^{40}$. It is still unclear why only a few patients will have a reoccurrence and what might help to predict and prevent it.

The ESMO Clinical Practice Guidelines consider the possibility to restart ICPI, as described in 5) above. The decision to stop life-saving therapies is not straight-forward and should be a matter of multidisciplinary discussion. Patients with higher AKI stages ( $\geq$ stage 2 ) should be referred to Nephrology consultation to evaluate the cause of renal dysfunction. A renal biopsy might differentiate an acute tubular necrosis (ATN) from a real irAE requiring ICPI suspension, CCT, or other immunosuppressors, while at the same time avoiding unnecessary toxicity or delays in treatment. The evidence presented by Izzedine et al. shows that ATN might account for a large part of kidney dysfunction ( 5 of 12 patients presented with ATN alone) ${ }^{42}$.

The safety of retreatment also depends on the severity of the irAE. A life-threatening toxicity should be regarded as an absolute contra-indication to resume immunotherapy ${ }^{6}$.

Another pertinent issue frequently raised is whether CCT or other immunosuppressors negatively impact the treatment of cancer. Current evidence shows that patients treated for irAEs did not have worse outcomes ${ }^{49-51}$. Some papers further suggest that irAEs correlate with a better cancer response ${ }^{51}$. 


\section{PATIENTS AT INCREASED RISK FOR ADVERSE EVENTS}

Patients with previous Al diseases are at increased risk for irAEs. Data on these patients are scarce since they have been excluded from most clinical trials. In the few retrospective studies that involved patients with a history of Al disease[52-54], 27-38\% had a flare. In the survey conducted by Menzies et al., only $4 \%$ ( 2 of 20 ) had to stop treatment for this reason ${ }^{52}$. Among the population with Al diseases in the study led by Johnson et al., all flares were successfully managed with $\mathrm{CCT}^{53}$. Danlos et al. also demonstrate that treatment with anti-PD1 was maintained in most patients with previous Al dysfunctions, despite irAEs, and cancer treatment was just as effective ${ }^{54}$. The relatively rare reports of a high-grade flare from an existing Al disease suggests that patients with a life-threatening cancer might be considered for immunotherapy after careful multidisciplinary discussion, pending close clinical and analytical vigilance ${ }^{6}$.

CTLA4 and PD1 are involved in immunologic mechanisms that enable transplanted organ tolerance. Interfering with these pathways raises a high risk of organ rejection. Transplanted patients have been excluded from clinical trials, and evidence comes from selected case reports. De Bruyn et al. describe $45 \%$ of renal allograft rejection in a cohort of 29 patients ${ }^{55}$. Four patients obtained a cancer response without organ rejection. Abdel-Wahab et al. describe 23 patients with a transplanted kidney treated with ICPI, $43 \%$ of whom lost their grafts $^{56}$. Four patients died from complications associated with rejection ( 2 of them had synchronous allograft rejection and disease progression). Barnett et al. report a case where immunosuppression was preemptively altered before ICPI treatment (CCT initiation and switch from tacrolimus to sirolimus) to help preserve the renal graft, with successful results ${ }^{57}$. However, Abdel-Wahab et al. point out 10 of 20 patients who, despite preemptive modifications of the baseline immunosuppression regimen, had a graft rejection ${ }^{56}$.

When it comes to transplanted patients with life-threatening cancer, the transplanted organ should be taken into consideration. Kidney failure might be treated with dialysis, whereas other organs failure might be more challenging to handle. Still, it should be decided on a case-by-case approach.

Patients with renal insufficiency were also excluded from most clinical trials. A prospective study with atezolizumab involved patients with a glomerular filtration rate between $30-60 \mathrm{ml} / \mathrm{min}$, who had a comparable response to therapy ${ }^{58}$. ICPI are not cleared by the kidneys, so they should be just as effective and safe for patients with chronic kidney disease. The findings of Kanz et al. corroborate this. The authors identified 17 patients on ICPI with renal dysfunction, including 3 on hemodialysis, that didn't have worse disease responses or more irAEs ${ }^{59}$. Herz et al. report another 4 patients with kidney failure, one of them with a kidney graft, that had stable renal function, and no irAE (or rejection) ${ }^{60}$.

\section{CONCLUSION}

ICPI represent a massive advance in the treatment of cancer, and their revolutionary results are being explored in a growing field of diseases and patients. Evidence shows that renal toxicities are probably much more common than initially reported. Up to this moment, there are no validated biomarkers for the prediction of ICPI toxicity, thus its management relies on an early diagnosis and high suspicion that should lead to a prompt and aggressive use of CCT or other immunosuppressors. Renal biopsy may play a crucial role. The multiple potential organ toxicities demand a multidisciplinary approach, and the nephrologists should be ready to take part in the diagnosis and treatment strategy.

\section{Disclosure of potential conflicts of interest: none declared}

\section{References}

1. Keung EZ, Ukponmwan EU, Cogdill AP, Wargo JA. The rationale and emerging use of neoadjuvant immune checkpoint blockade for solid malignancies. Ann Surg Oncol 2018;25:1814-27. doi:10.1245/s10434-018-6379-8.

2. Cortazar FB, Marrone KA, Troxell ML, Ralto KM, Hoenig MP, Brahmer JR, et al. Clinicopathological features of acute kidney injury associated with immune checkpoint inhibitors HHS Public Access. Kidney Int 2016;90:638-47. doi:10.1016/j.kint.2016.04.008.

3. Amos SM, Duong CPM, Westwood JA, Ritchie DS, Junghans RP, Darcy †phillip K, et al. Autoimmunity associated with immunotherapy of cancer 2011. doi:10.1182/blood-2011-01-325266.

4. Krummel ME, Allison JP. CTLA-4 Engagement Inhibits 11.+-2 Accumulation and Cell Cycle Progression upon Activation of Resting T Cells. n.d.

5. Boussiotis VA. Molecular and Biochemical Aspects of the PD-1 Checkpoint Pathway IMMUNE CHECKPOINT BLOCKADE AS CANCER THERAPY n.d. doi:10.1056/NEJMra1514296.

6. Postow MA, Sidlow R, Hellmann MD. Immune-related adverse events associated with immune checkpoint blockade. N Engl J Med 2018;378:158-68. doi:10.1056/NEJMra1703481.

7. Sibaud V. Dermatologic Reactions to immune checkpoint inhibitors. Am J Clin Dermatol 2018;19:345-61. doi:10.1007/s40257-017-0336-3.

8. Som A, Mandaliya R, Alsaadi D, Farshidpour M, Charabaty A, Malhotra N, et al. Immune checkpoint inhibitor-induced colitis: A comprehensive review. World J Clin Cases 2019;7:405-18. doi:10.12998/ wjcc.v7.i4.405.

9. Girotra M, Hansen A, Farooki A, Byun DJ, Min L, Creelan BC, et al. The current understanding of the endocrine effects from immune checkpoint inhibitors and recommendations for management. JNCl Cancer Spectr 2018;2:pky021. doi:10.1093/jncics/pky021.

10. Waterhouse P, Penninger JM, Timms E, Wakeham A, Shahinian A, Lee KP, et al. Lymphoproliferative disorders with early lethality in mice deficient in Ctla-4. Science (80-) 1995;270:985-8. doi:10.1126/science.270.5238.985.

11. Nishimura $\mathrm{H}$, Nose $\mathrm{M}$, Hiai $\mathrm{H}$, Minato $\mathrm{N}$, Honjo T. Development of lupus-like autoimmune diseases by disruption of the PD-1 gene encoding an ITIM motif-carrying immunoreceptor. Immunity 1999;11:141-51. doi:10.1016/S1074-7613(00)80089-8.

12. Vital EM, Emery P. Abatacept in the treatment of rheumatoid arthritis. Ther Clin Risk Manag 2006;2:365.

13. Noisette A, Hochberg MC. Abatacept for the treatment of adults with psoriatic arthritis: patient selection and perspectives. Psoriasis (Auckland, NZ) 2018;8:31-9. doi:10.2147/PTT.S146076.

14. Pimentel-Quiroz VR, Ugarte-Gil MF, Alarcón GS. Abatacept for the treatment of systemic lupus erythematosus. Expert Opin Investig Drugs 2016;25:493-9. doi:10.1517/13543784.2016.1154943.

15. Petrella TM, Robert C, Richtig E, Miller WH, Masucci G V., Walpole E, et al. Patient-reported outcomes in KEYNOTE-006, a randomised study of pembrolizumab versus ipilimumab in patients with advanced melanoma. Eur J Cancer 2017;86:115-24. doi:10.1016/J.EJCA.2017.08.032.

16. Michot JM, Bigenwald C, Champiat S, Collins M, Carbonnel F, Postel-Vinay S, et al. Immune-related adverse events with immune checkpoint blockade: a comprehensive review. Eur J Cancer 2016;54:139-48. doi:10.1016/j.ejca.2015.11.016.

17. Wolchok JD, Chiarion-Sileni V, Gonzalez R, Rutkowski P, Grob J-J, Cowey CL, et al. Overall survival with combined nivolumab and ipilimumab in advanced melanoma. N Engl J Med 2017;377:134556. doi:10.1056/NEJMoa1709684.

18. Larkin J, Chiarion-Sileni V, Gonzalez R, Grob J-J, Rutkowski P, Lao CD, et al. Five-year survival with combined nivolumab and ipilimumab in advanced melanoma. N Engl J Med 2019. doi:10.1056/ nejmoa1910836.

19. Byrne EH, Fisher DE. Immune and molecular correlates in melanoma treated with immune checkpoint blockade. Cancer 2017;123:2143-53. doi:10.1002/cncr.30444.

20. Fife BT, Pauken KE. The role of the PD-1 pathway in autoimmunity and peripheral tolerance. Ann N Y Acad Sci 2011;1217:45-59. doi:10.1111/j.1749-6632.2010.05919.x.

21. Callahan MK, Yang A, Tandon S, Xu Y, Subudhi SK, Roman RA, et al. Evaluation of serum IL-17 levels during ipilimumab therapy: correlation with colitis. J Clin Oncol 2011;29:2505-2505. doi:10.1200/jco.2011.29.15_suppl.2505.

22. Mamlouk O, Selamet U, Machado S, Abdelrahim M, Glass WF, Tchakarov A, et al. Nephrotoxicity of immune checkpoint inhibitors beyond tubulointerstitial nephritis: single-center experience. J Immunother Cancer 2019;7. doi:10.1186/s40425-018-0478-8.

23. Wanchoo R, Karam S, Uppal NN, Barta VS, Deray G, Devoe C, et al. Adverse renal effects of immune checkpoint inhibitors: a narrative review. Am J Nephrol 2017:45:160-9. doi:10.1159/000455014. 
24. Launay-Vacher V. Epidemiology of chronic kidney disease in cancer patients: lessons from the IRMA study group. Semin Nephrol 2010;30:548-56. doi:10.1016/j.semnephrol.2010.09.003.

25. Launay-Vacher V, Oudard S, Janus N, Gligorov J, Pourrat X, Rixe O, et al. Prevalence of renal insufficiency in cancer patients and implications for anticancer drug management. Cancer 2007;110:1376-84. doi:10.1002/cncr.22904.

26. Gallan AJ, Alexander E, Reid P, Kutuby F, Chang A, Henriksen KJ. Renal vasculitis and pauci-immune glomerulonephritis associated With immune checkpoint inhibitors. Am J Kidney Dis 2019:1-4. doi:10.1053/j.ajkd.2019.04.016.

27. Cassol C, Satoskar A, Lozanski G, Rovin B, Hebert L, Nadasdy T, et al. AntiLPD-1 immunotherapy may induce interstitial nephritis with increased tubular epithelial expression of PD-L1 2019. doi:10.1016/j.ekir.2019.06.001.

28. Perazella MA, Markowitz GS. Drug-induced acute interstitial nephritis. Nat Rev Nephrol 2010;6:461-70. doi:10.1038/nrneph.2010.71

29. Koda R, Watanabe H, Tsuchida M, lino N, Suzuki K, Imai N, et al. Immune checkpoint inhibito (nivolumab)-associated kidney injury and the importance of recognizing concomitant medications known to cause acute tubulointerstitial nephritis: a case report n.d. doi:10.1186/s12882-018-0848-y.

30. Nakatani Y, Kawakami H, Ichikawa M, Yamamoto S, Otsuka Y, Mashiko A, et al. Nivolumab-induced acute granulomatous tubulointerstitial nephritis in a patient with gastric cancer. Invest New Drugs 2018:36:726-31. doi:10.1007/s10637-018-0596-7.

31. Izzedine H, Mateus C, Boutros C, Robert C, Rouvier P, Amoura Z, et al. Renal effects of immune checkpoint inhibitors. Nephrol Dial Transplant 2017;32:936-42. doi:10.1093/ndt/gfw382.

32. Bottlaender L, Breton AL, Laforcade L, Dijoud F, Thomas L, Dalle S. Acute interstitial nephritis after sequential ipilumumab - nivolumab therapy of metastatic melanoma. J Immunother Cancer 2017;5. doi:10.1186/s40425-017-0261-2.

33. Basnet $S$, Dhital R, Tharu B. medicina Acute tubulointerstitial nephritis: a case report on rare adverse effect of pembrolizumab n.d. doi:10.3390/medicina55050176.

34. Tabei A, Watanabe M, Ikeuchi H, Nakasatomi M, Sakairi T, Kaneko Y, et al. The analysis of renal infiltrating cells in acute tubulointerstitial nephritis induced by anti-PD-1 antibodies: a case report and review of the literature. Intern Med 2018;57:3135-9. doi:10.2169/internalmedicine.0444-17.

35. Glutsch V, Grän F, Weber J, Gesierich A, Goebeler M, Schilling B. Response to combined ipilimumab and nivolumab after development of a nephrotic syndrome related to PD-1 monotherapy. J Immunother Cancer 2019;7. doi:10.1186/s40425-019-0655-4.

36. Fadel F, Karoui K El, Knebelmann B. Anti-CTLA4 Antibody-induced lupus nephritis. N Engl J Med 2009;361:211-2. doi:10.1056/NEJMc0904283.

37. Daanen RA, Maas RJH, Koornstra RHT, Steenbergen EJ, Van Herpen CML, Willemsen AECAB. Nivolumab-associated nephrotic syndrome in a patient with renal cell carcinoma: a case report. J Immunother 2017;40:345-8. doi:10.1097/CJI.00000000000000189.

38. Jung K, Zeng X, Bilusic M. Nivolumab-associated acute glomerulonephritis: a case report and literature review. n.d. doi:10.1186/s12882-016-0408-2.

39. van den Brom RRH, Abdulahad WH, Rutgers A, Kroesen B-J, Roozendaal C, de Groot DJA, et al. Rapid granulomatosis with polyangiitis induced by immune checkpoint inhibition. Rheumatology 2016;55:1143-5. doi:10.1093/rheumatology/kew063.

40. Kitchlu A, Fingrut W, Avila-Casado C, Chan CT, Crump M, Hogg D, et al. Nephrotic syndrome with cancer immunotherapies: a report of 2 cases. Am J Kidney Dis 2017;70:581-5. doi:10.1053/j. ajkd.2017.04.026

41. Kishi S, Minato M, Saijo A, Murakami N, Tamaki M, Matsuura M, et al. IgA nephropathy after nivolumab therapy for postoperative recurrence of lung squamous cell carcinoma. Intern Med 2018;57:1259-63. doi:10.2169/internalmedicine.9814-17.

42. Izzedine H, Mathian A, Champiat S, Picard C, Mateus C, Routier E, et al. Renal toxicities associated with pembrolizumab. Clin Kidney J 2019;12:81-8. doi:10.1093/ckj/sfy100.

43. Barnard ZR, Walcott BP, Kahle KT, Nahed B V., Coumans JV. Hyponatremia associated with Ipilimumab-induced hypophysitis. Med Oncol 2012;29:374-7. doi:10.1007/s12032-010-9794-7.

44. Manohar S, Kompotiatis P, Thongprayoon C, Cheungpasitporn W, Herrmann J, Herrmann SM. Programmed cell death protein 1 inhibitor treatment is associated with acute kidney injury and hypocalcemia: meta-analysis. Nephrol Dial Transplant 2019;34:108-17. doi:10.1093/ndt/gfy105.

45. Haanen JBAG, Carbonnel F, Robert C, Kerr KM, Peters S, Larkin J, et al. Management of toxicities from immunotherapy: ESMO Clinical Practice Guidelines for diagnosis, treatment and follow-upt. Ann Oncol 2017;28:iv119-42. doi:10.1093/annonc/mdx225.
46. Brahmer JR, Lacchetti C, Schneider BJ, Atkins MB, Brassil KJ, Caterino JM, et al. Management of immune-related adverse events in patients treated with immune checkpoint inhibitor therapy: american society of clinical oncology clinical practice guideline. J Clin Oncol 2018;36:1714-68. doi:10.1200/JCO.2017.77.6385.

47. Preddie DC, Markowitz GS, Radhakrishnan J, Nickolas TL, D’Agati VD, Schwimmer JA, et al. Mycophenolate mofetil for the treatment of interstitial nephritis. Clin J Am Soc Nephrol 2006;1:718-22. doi:10.2215/CJN.01711105.

48. Santini FC, Rizvi H, Plodkowski AJ, Ni A, Lacouture ME, Gambarin-Gelwan M, et al. Safety and efficacy of re-treating with immunotherapy after immune-related adverse events in patients with NSCLC. Cancer Immunol Res 2018;6:1093-9. doi:10.1158/2326-6066.CIR-17-0755.

49. Weber JS, Hodi FS, Wolchok JD, Topalian SL, Schadendorf D, Larkin J, et al. Safety profile of nivolumab monotherapy: a pooled analysis of patients with advanced melanoma. J Clin Oncol 2017:35:785-92. doi:10.1200/JCO.2015.66.1389.

50. Horvat TZ, Adel NG, Dang T-O, Momtaz P, Postow MA, Callahan MK, et al. Immune-related adverse events, need for systemic immunosuppression, and effects on survival and time to treatment failure in patients With melanoma treated with Ipilimumab at Memorial Sloan Kettering Cancer Center. J Clin Oncol 2015;33:3193-8. doi:10.1200/JCO.2015.60.8448.

51. Weber JS, Hodi FS, Wolchok JD, Topalian SL, Schadendorf D, Larkin J, et al. Safety profile of nivolumab monotherapy: a pooled analysis of patients with advanced melanoma. J Clin Oncol 2017:35:785-92. doi:10.1200/JCO.2015.66.1389.

52. Menzies AM, Johnson DB, Ramanujam S, Atkinson VG, Wong ANM, Park JJ, et al. Anti-PD-1 therapy in patients with advanced melanoma and preexisting autoimmune disorders or major toxicity with ipilimumab. Ann Oncol 2016;28:mdw443. doi:10.1093/annonc/mdw443.

53. Johnson DB, Sullivan RJ, Ott PA, Carlino MS, Khushalani NI, Ye F, et al. Ipilimumab therapy in patients with advanced melanoma and preexisting autoimmune disorders. JAMA Oncol 2016;2:234. doi:10.1001/jamaoncol.2015.4368.

54. Danlos F-X, Voisin A-L, Dyevre V, Michot J-M, Routier E, Taillade L, et al. Safety and efficacy of anti-programmed death 1 antibodies in patients with cancer and pre-existing autoimmune or inflammatory disease. Eur J Cancer 2018;91:21-9. doi:10.1016/j.ejca.2017.12.008.

55. De Bruyn P, Van Gestel D, Ost P, Kruse V, Brochez L, Van Vlierberghe H, et al. Immune checkpoint blockade for organ transplant patients with advanced cancer: how far can we go? Curr Opin Oncol 2019;31:54-64. doi:10.1097/CCO.0000000000000505

56. Abdel-Wahab N, Safa H, Abudayyeh A, Johnson DH, Trinh VA, Zobniw CM, et al. Checkpoint inhibitor therapy for cancer in solid organ transplantation recipients: an institutional experience and a systematic review of the literature. J Immunother Cancer 2019;7. doi:10.1186/s40425-019$-0585-1$.

57. Barnett R, Barta VS, Jhaveri KD. Preserved renal-allograft function and the PD-1 pathway inhibitor nivolumab: to the editor. N Engl J Med 2017;376:191-2. doi:10.1056/NEJMc1614298.

58. Balar A V., Galsky MD, Rosenberg JE, Powles T, Petrylak DP, Bellmunt J, et al. Atezolizumab as first-line treatment in cisplatin-ineligible patients with locally advanced and metastatic urothelia carcinoma: a single-arm, multicentre, phase 2 trial. Lancet 2017;389:67-76. doi:10.1016/50140$-6736(16) 32455-2$

59. Kanz BA, Pollack MH, Johnpulle R, Puzanov I, Horn L, Morgans A, et al. Safety and efficacy of anti-PD-1 in patients with baseline cardiac, renal, or hepatic dysfunction. J Immunother Cancer 2016;4. doi:10.1186/s40425-016-0166-5.

60. Herz S, Höfer T, Papapanagiotou M, Leyh JC, Meyenburg S, Schadendorf D, et al. Checkpoint inhibitors in chronic kidney failure and an organ transplant recipient. Eur J Cancer 2016;67:66-72. doi:10.1016/j.ejca.2016.07.026

\section{Correspondence to:}

\section{Teresa Chuva}

Nephrology Department, Instituto Português de Oncologia do Porto E-mail: m.teresa.chuva@gmail.com 